\title{
Improved Clinical Outcomes and Patient Satisfaction of In-Office Needle Arthroscopy for the Treatment of Posterior Ankle Impingement
}

\author{
Nathaniel P. Mercer, M.S., Alan P. Samsonov, B.S., John F. Dankert, M.D., Ph.D., \\ Arianna L. Gianakos, D.O., Tobias Stornebrink, M.D., Rick J. Delmonte, D.P.M., \\ Gino M. M. J. Kerkhoffs, M.D., Ph.D., and \\ John G. Kennedy, M.D., M.Ch., M.M.Sc., F.F.S.E.M., F.R.C.S. (Orth.)
}

\begin{abstract}
Purpose: To investigate the short-term clinical outcomes and satisfaction for the first set of patients at our institution receiving in-office needle arthroscopy (IONA) for the treatment of posterior ankle impingement syndrome (PAIS). Methods: A retrospective cohort study was conducted to evaluate patients who underwent IONA for PAIS between January 2019 and January 2021. Clinical outcomes were evaluated using the Foot and Ankle Outcome Score (FAOS) and Patient-Reported Outcomes Measurement Information System Pain Interference, and Pain Intensity scores. Patient satisfaction was measured at the final follow-up visit with a 5-point Likert scale. The Wilcoxon signed-rank test was performed to compare preoperative and postoperative outcome scores. Results: Ten patients (4 male and 6 female) with a mean age of $41.9 \pm 15.5$ years (range, 24-66 years) were included in the study. The mean follow-up time was $13.3 \pm 2.9$ months (range, 11-17 months). All mean preoperative FAOS scores demonstrated improvement after IONA, including FAOS symptoms $(71.48 \pm 10.3$ to $80.3 \pm 12.6)$, pain $(69.3 \pm 11.0$ to $78.2 \pm 13.9)$, activities of daily living $(61.7 \pm 8.8$ to $77.93 \pm 11.4)$, sports activities $(55.6 \pm 12.7$ to $76.0 \pm 13.6)$, and quality of life $(46.6 \pm 9.2$ to $71.1 \pm 12.1)$. There were 7 patients who participated in sports activities before the IONA procedure. Within this group, all patients returned to play at a median time of 4.1 weeks (range, 1-14 weeks). The median time to return to work was $3.4 \pm 5.3$ days. Patients reported an overall positive IONA experience with a mean rating scale of $9.5 \pm 1.5$ (range, 5-10). Conclusions: The current study demonstrates that IONA treatment of PAIS results in significant pain reduction, a low complication rate, and excellent patient-reported outcomes. In addition, IONA for PAIS leads to high patient satisfaction with a significant willingness to undergo the same procedure again. Level of Evidence: IV, therapeutic case series.
\end{abstract}

$\mathbf{P}$ osterior ankle impingement syndrome (PAIS) is a syndrome involving posterior hindfoot pain due to the impingement of the posterior ankle joint. PAIS can be debilitating, especially in athletes enduring repeated plantarflexion, like ballet dancers, soccer players, and

From the Department of Orthopedic Surgery, NYU Langone Health, New York, New York, U.S.A. (N.P.M., A.P.S., J.F.D., R.J.D., J.G.K.); Department of Orthopedic Surgery, Amsterdam Movement Sciences, Amsterdam UMC, University of Amsterdam, Amsterdam, The Netherlands (T.S., G.M.M.J.K.); Academic Center for Evidence-based Sports Medicine (ACES), Amsterdam, The Netherlands (T.S., G.M.M.J.K.); Amsterdam Collaboration for Health and Safety in Sports (ACHSS), International Olympic Committee (IOC) Research Center Amsterdam UMC, Amsterdam, The Netherlands (T.S., G.M.M.J.K.); and Harvard - Massachusetts General Hospital, Boston, Massachusetts, U.S.A. (A.L.G.).

The authors report the following potential conflicts of interest or sources of funding: G.M.M.J.K. reports board or committee member, European Society of Sports Traumatology, Knee Surgery and Arthroscopy. J.G.K. received support from the Ohnell Family Foundation and Mr. and Mrs. Michael J. Levitt. He is a consultant for Arteriocyte Industries (Isto Biologics) and Arthrex, outside the downhill runners. ${ }^{1}$ Several factors may contribute to the development of PAIS, including but not limited to variations in soft tissue and bony anatomy, such as an os trigonum or Stieda process. ${ }^{2}$ With repetitive plantarflexion, soft or bony tissue may become compressed,

submitted work, and reports board or committee member, American Orthopaedic Foot and Ankle Society, European Society of Sports Traumatology, Knee Surgery and Arthroscopy, Ankle and Foot Associates, and International Society for Cartilage Repair of the Ankle. Full ICMJE author disclosure forms are available for this article online, as supplementary material.

Received April 4, 2021; accepted December 1, 2021.

Address correspondence to John G. Kennedy, M.D., M.Ch., M.M.Sc., F.F.S.E.M., F.R.C.S. (Orth.), 171 Delancey St., New York, NY 10002. E-mail: John.Kennedy@nyulangone.org

(C) 2022 Published by Elsevier Inc. on behalf of the Arthroscopy Association of North America. This is an open access article under the CC BY-NC-ND license (http://creativecommons.org/licenses/by-nc-nd/4.0/).

2666-061X/21480

https://doi.org/10.1016/j.asmr.2021.12.004 
resulting in inflammation and pain. Both conservative and surgical options are available for PAIS, and while conservative management may afford temporizing relief, surgery is often required. While studies have demonstrated that open procedures result in good clinical outcomes, most posterior ankle debridement is now performed using standard arthroscopy, with excellent outcomes and shortened times to return to play for athletes. ${ }^{1,3-6}$

In-office needle endoscopy or in-office needle arthroscopy (IONA) first became available in the 1990s as a technique to visualize and evaluate joints under local anesthesia. ${ }^{7}$ These systems never achieved widespread adoption in part due to poor picture quality and unclear indications for their use. Furthermore, no instruments were available to intervene on identified pathology, which prevented IONA from becoming more than a diagnostic tool. IONA recently has undergone a technological advancement with the release of a disposable chipon-tip camera with $400 \times 400$-pixel resolution and $120^{\circ}$ field of view fed through a $13^{\prime \prime}$ high-definition monitor. Importantly, this new system now has instrumentation including punches, graspers, scissors, a retractable probe, shavers, burrs, and resectors available for concomitant intervention during IONA. Using IONA instead of traditional arthroscopy provides patients the opportunity to avoid the costs of anesthesia and a full operating suite. The timing of the release of the next-generation IONA was just before the coronavirus disease 2019 (COVID-19) pandemic in the United States. As more and more patients became reluctant to go to major hospital facilities for fear of being exposed to COVID-19, the ability to perform the same surgery in an outpatient setting became more desirable. Hospitals formulated guidelines for nonemergent medical care, decreasing the number of elective procedures in the peripandemic period. ${ }^{8}$ Consequently, this shift in patient perception of safety allowed the investigators a unique opportunity to evaluate the use of IONA on select pathology over a defined short period of time and compare outcomes with traditional arthroscopic procedures performed in an operating room.

The purpose of this study was to investigate the shortterm clinical outcomes and satisfaction for the first set of patients at our institution receiving IONA for the treatment of PAIS. We hypothesized that patients would have improved overall satisfaction from the procedure due to their interaction with the surgical team, with clinical outcomes at least similar to the published results for PAIS when managed with traditional arthroscopy.

\section{Methods}

\section{Patients}

After approval from our institutional review board, a prospectively collected database of 10 patients undergoing IONA for the treatment of PAIS between January 2019 and January 2020 was retrospectively reviewed. Indication for IONA treatment was a diagnosis of PAIS that was refractory to conservative treatment measures for greater than 3 months. Conservative treatment measures consisted of rest, modification of activity, physiotherapy, anti-inflammatory drugs, and ultrasound-guided injections. Inclusion criteria for this study were patients $\geq 18$ years of age and clinical history, physical history, radiographic imaging, and magnetic resonance imaging consistent with PAIS for which each patient underwent IONA and had a minimum of 12-month follow-up. Only primary IONA for PAIS were included and all patients who had concomitant procedures other than debridement for PAIS were excluded.

Patients were diagnosed with PAIS based on a combination of physical examination and radiographic findings. In the clinical setting, PAIS is characterized by a deep posterior ankle pain caused by plantar flexion of the ankle joint, which indicates a positive plantar flexion test. ${ }^{9}$ Patients also may demonstrate tenderness over the posteromedial aspect of the ankle joint. To further specify the symptom location, the clinician may passively flex and extend the great toe, and if the patient elicits pain during passive or active range of motion, it may indicate pathology involving the flexor hallucis longus tendon. ${ }^{1}$ If clinical examination findings are positive, the authors prefer to evaluate the condition of hindfoot structures using plain radiographs (anteroposterior, mortise, and lateral view) and magnetic resonance imaging. Then, an ultrasound diagnostic injection using a local anesthetic used to confirm diagnosis. After nonresponse to conservative treatment, and subsequent decision to treat with IONA, all patients underwent final assessment in the office under direct visualization for signs of PAIS with a needle arthroscope.

\section{Data Collection}

Data on patient characteristics and clinical information were collected. Clinical outcomes were evaluated using the Foot and Ankle Outcomes Score (FAOS) and Patient-Reported Outcomes Measurement Information System (PROMIS) Pain Interference and Pain Intensity scores preoperatively and at the final follow-up visit. Patient satisfaction with the IONA procedure was evaluated by a 5 -point Likert scale at the final follow-up visit. The following questionnaire was used to evaluate patient experience after undergoing in-office needle arthroscopy: How was your overall experience seeing your underlying pathology at the time of the procedure (rating scale: 0 negative experience -5 neutral -10 positive experience)?; Do you feel this experience aided in your understanding of posterior ankle impingement (rating scale: 0 negative experience -5 neutral -10 
Table 1. Overall Clinical Outcome Scores for Posterior IONA $(\mathrm{n}=10)$

\begin{tabular}{|c|c|c|c|c|c|}
\hline \multirow[b]{2}{*}{ Patient } & \multirow[b]{2}{*}{ Sport } & \multicolumn{2}{|c|}{ Preoperative Score } & \multicolumn{2}{|c|}{ Postoperative Score } \\
\hline & & FAOS & PROMIS & FAOS & PROMIS \\
\hline 1 & Golf & $52.2,50.3,65,35,45$ & $52.3,65.7$ & $46.4,55.6,64.7,40,60$ & $49.9,62.6$ \\
\hline 2 & Running & $70.75,75.545,40,30$ & $54.4,65.7$ & $85.7,91.7,70.6,80,75$ & $51.3,56$ \\
\hline 3 & Hockey & $78.8,70,60,45,56.2$ & $50.5,72.2$ & $89.3,83,90,85,63$ & $40.5,56$ \\
\hline 4 & Walking & $79.5,52.3,65,50.5,50.3$ & $60.6,70.5$ & $82.1,58.3,94.1,80,94$ & $48.4,61.6$ \\
\hline 5 & None & $85,80.4,60,50,44.3$ & $68.3,76.5$ & $96,94.2,70.6,80,56.3$ & $49.9,73.3$ \\
\hline 6 & Hiking & $72.5,74.7,55,65,36.3$ & $42.6,54.4$ & $82.1,94.4,72,75,68.8$ & $40.5,60.3$ \\
\hline 7 & None & $75,75,67.4,70,44.8$ & $71.8,73.7$ & $78.6,72,91,85,56.3$ & $60.6,66.9$ \\
\hline 8 & None & $65,60,55,75,56.7$ & $52.2,68.4$ & $82.1,70.6,70.6,65,75$ & $52.3,66.9$ \\
\hline 9 & Running & $80.5,85,80,60,61.2$ & $62.1,71.6$ & $86.4,91,91,80,75$ & $52.3,65.7$ \\
\hline 10 & Golf & $55.8,70.2,65,65,41.2$ & $60.6,71.6$ & $75,70.6,64.7,90,87.5$ & $49.9,59.1$ \\
\hline
\end{tabular}

NOTE. FAOS includes symptoms, pain, ADL, sports, QOL and PROMIS Pain Interference, Pain Intensity.

ADL, activities of daily living; FAOS, Foot and Ankle Outcome Score; IONA, in-office needle arthroscopy; PROMIS, Patient-Reported Outcomes Measurement Information System; QOL, quality of life.

positive experience)?; Would you prefer having this same procedure in the office, operating room, or do you have no preference (rating scale: 0 operating room -5 neutral - 10 office)?; Did you experience any pain during the procedure (rating scale: 0 is no pain, 10 is worst pain)?; Would you undergo the same procedure again (Yes or No)? The minimal clinically important difference (MCID) represents the smallest change in pre- and postoperative patient-reported outcome scores that show a clinically significant difference in symptom improvement or worsening. MCID was calculated for FAOS Symptoms, FAOS Pain, FAOS activities of daily living, FAOS sports, FAOS quality of life, and PROMIS Pain interference and PROMIS Pain intensity using the most common and well-described distribution-based method of standard deviation (SD): MCID = $0.5^{*} \mathrm{SD} \cdot{ }^{10,11}$ Patients' individual preoperative and postoperative functional outcome scores were individually reported and are listed in Table 1 . In addition, for all functional outcome measures, achievement of MCID was recorded and listed in Table 2.

\section{Statistical Analysis}

Descriptive statistics were completed consisting of mean and SD for continuous variables and frequency and percentage for categorical variables. A Wilcoxon signed-rank test was performed to compare preoperative and postoperative outcome scores. A value of $P<$ .05 was considered statistically significant. All statistical analysis was performed with SPSS, version 22 (IBM Corp. Released 2013; IBM SPSS Statistics for Windows, Armonk, NY.)

\section{Operative Technique}

The patient is positioned on an examination table in the prone position with the foot over the edge of the bed. If the patient is interested in observing the procedure, then a mirror may be placed at the head of the bed allowing him or her to view of the procedure and video monitor. A standard setup of the IONA can be seen in Figure 1 and Figure 2. If difficulty with joint access is anticipated, traction options should be available, including a traditional ankle stirrup or manual

Table 2. MCID for Clinical Outcomes $(n=10)$

\begin{tabular}{|c|c|c|c|c|c|c|c|c|}
\hline Patient & Sport & $\begin{array}{c}\text { Pain Interference } \\
\text { PROMIS Achieve } \\
\text { MCID (Y/N) }\end{array}$ & $\begin{array}{c}\text { Pain Intensity } \\
\text { PROMIS Achieve } \\
\text { MCID (Y/N) }\end{array}$ & $\begin{array}{c}\text { FAOS Symptoms } \\
\text { Achieve MCID } \\
(\mathrm{Y} / \mathrm{N})\end{array}$ & $\begin{array}{c}\text { FAOS Pain } \\
\text { Achieve MCID } \\
(\mathrm{Y} / \mathrm{N}) \\
\end{array}$ & $\begin{array}{c}\text { FAOS ADL } \\
\text { Achieve MCID } \\
(\mathrm{Y} / \mathrm{N}) \\
\end{array}$ & $\begin{array}{c}\text { FAOS Sports } \\
\text { Achieve MCID } \\
(\mathrm{Y} / \mathrm{N}) \\
\end{array}$ & $\begin{array}{c}\text { FAOS QOL } \\
\text { Achieve MCID } \\
(\mathrm{Y} / \mathrm{N})\end{array}$ \\
\hline 1 & Golf & $\mathrm{N}$ & $\mathrm{Y}$ & $\mathrm{N}$ & $\mathrm{N}$ & $\mathrm{N}$ & $\mathrm{N}$ & $\mathrm{Y}$ \\
\hline 3 & Hockey & $\mathrm{Y}$ & $\mathrm{Y}$ & $\mathrm{Y}$ & $\mathrm{Y}$ & $\mathrm{Y}$ & $\mathrm{Y}$ & $\mathrm{N}$ \\
\hline 4 & Walking & $\mathrm{Y}$ & $\mathrm{Y}$ & $\mathrm{N}$ & $\mathrm{Y}$ & $\mathrm{Y}$ & $\mathrm{Y}$ & $\mathrm{Y}$ \\
\hline 5 & None & $\mathrm{Y}$ & $\mathrm{Y}$ & $\mathrm{Y}$ & $\mathrm{Y}$ & $\mathrm{Y}$ & $\mathrm{Y}$ & $\mathrm{Y}$ \\
\hline 8 & None & $\mathrm{N}$ & $\mathrm{Y}$ & $\mathrm{Y}$ & $\mathrm{Y}$ & $\mathrm{Y}$ & $\mathrm{N}$ & $\mathrm{Y}$ \\
\hline 9 & Running & $\mathrm{Y}$ & $\mathrm{Y}$ & $\mathrm{Y}$ & $\mathrm{Y}$ & $\mathrm{Y}$ & $\mathrm{Y}$ & $\mathrm{Y}$ \\
\hline \multirow[t]{2}{*}{10} & Golf & $\mathrm{Y}$ & $\mathrm{Y}$ & $\mathrm{Y}$ & $\mathrm{N}$ & $\mathrm{N}$ & $\mathrm{Y}$ & $\mathrm{Y}$ \\
\hline & & $6 / 10(60 \%)$ & $9 / 10(90 \%)$ & $7 / 10(70 \%)$ & $7 / 10(70 \%)$ & $8 / 10(80 \%)$ & $8 / 10(80 \%)$ & $9 / 10(90 \%)$ \\
\hline
\end{tabular}

ADL, activities of daily living; FAOS, Foot and Ankle Outcome Score; MCID, minimum clinically important difference; N, no; PROMIS, PatientReported Outcomes Measurement Information System; QOL, quality of life; Y, yes. 


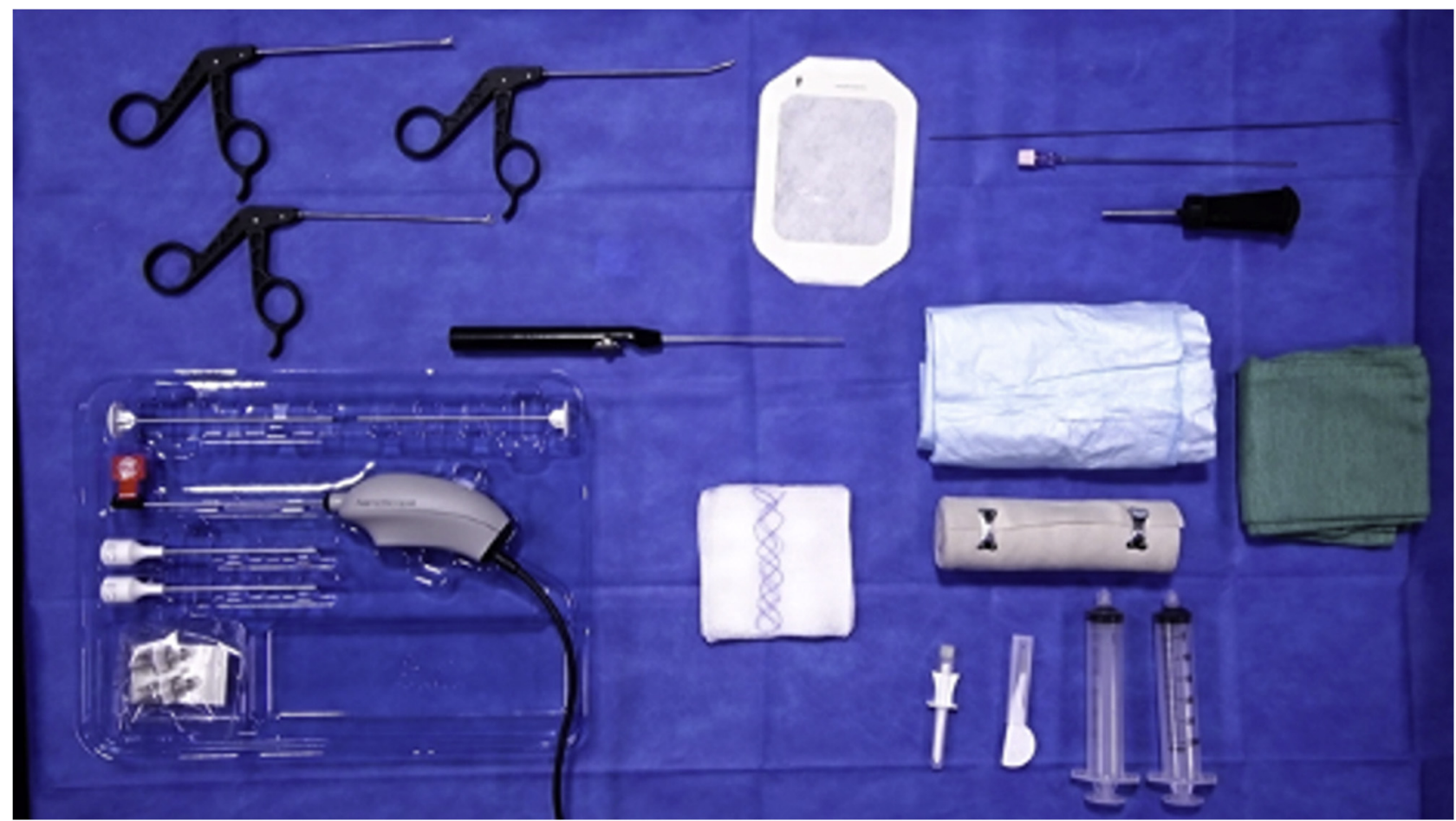

Fig 1. The equipment for the procedure is organized on a Mayo stand, which is draped in a sterile fashion and on which the equipment for the procedure is organized.

traction by an assistant. If using a stirrup, it is vital that the straps are secure and out of the way of anticipated portals.

The planned posterolateral and posteromedial arthroscopy portal sites were marked. The portal sites are $1.0 \mathrm{~mm}$ anterior to the borders of Achilles tendon and at the level of the transverse plane running from the inferior poles of the malleoli (Fig 3). The sural nerve can be palpated and its course marked to prevent iatrogenic nerve injury. Each site was injected with $1 \%$ lidocaine. A local block consisting of $15 \mathrm{~mL}$ of $2 \%$ lidocaine and $0.5 \%$ bupivacaine in a $1: 1$ ratio was delivered to the hindfoot via the anticipated portals. Using a \# 11 blade, the posterolateral portal was established. Blunt dissection was performed subcutaneously using a mosquito clamp with care taken to avoid the sural nerve. A cannula was then inserted into the portal in the direction of the third metatarsal to avoid medial neurovascular injury. A 1.9-mm $0^{\circ}$ needle arthroscope (NanoScope; Arthrex, Naples, FL) was placed through the cannula to visualize the posterior tibiotalar joint. A pump was set at $35 \mathrm{~mm} \mathrm{Hg}$ and saline with 1;100,000 adrenalin 1 cc was added. No tourniquet was used due to the minimal blood loss associated with the procedure.

The posteromedial portal was created under direct visualization in a similar fashion to the posterolateral portal. A $2.0-\mathrm{mm}$ shaver was introduced into the portal and careful debridement of fatty tissue was conducted until the posterior structures were all visualized. Care was taken during initial debridement and throughout the procedure to avoid iatrogenic injury. Debridement was carried out until the intermalleolar ligament (IML) was visualized.

Due to fatty or fibrotic tissue on the posterior portion of the ankle, it may be difficult to use arthroscopy in the approach to treat PAIS; therefore, we elect to perform this procedure in a systematic fashion by dividing the hindfoot into four quadrants (Fig 4). ${ }^{4,9}$ We have also listed some of the relevant pearls and pitfalls of this

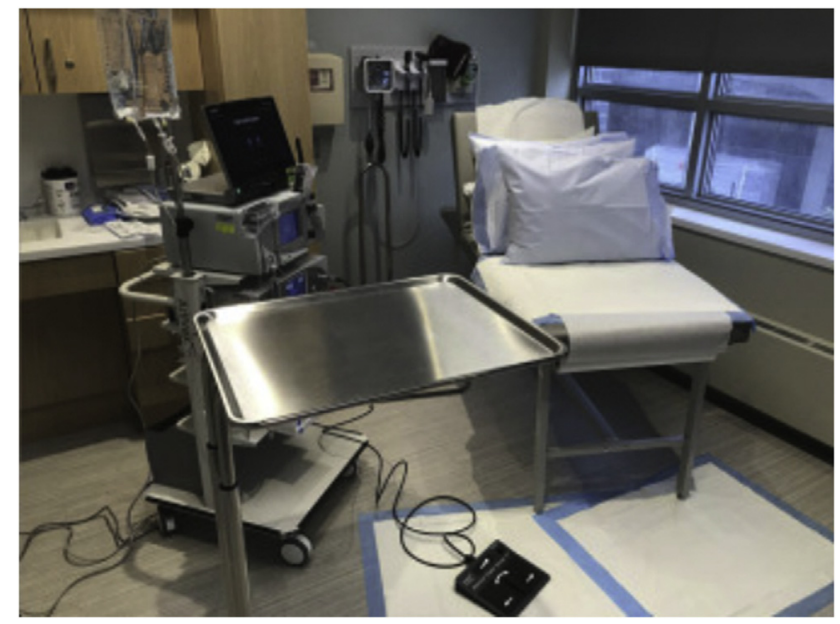

Fig 2. In-office needle arthroscopy standard setup. 
Fig 3. Relevant preoperative surface anatomy markings and portal locations are indicated on a posterolateral view of the left ankle.

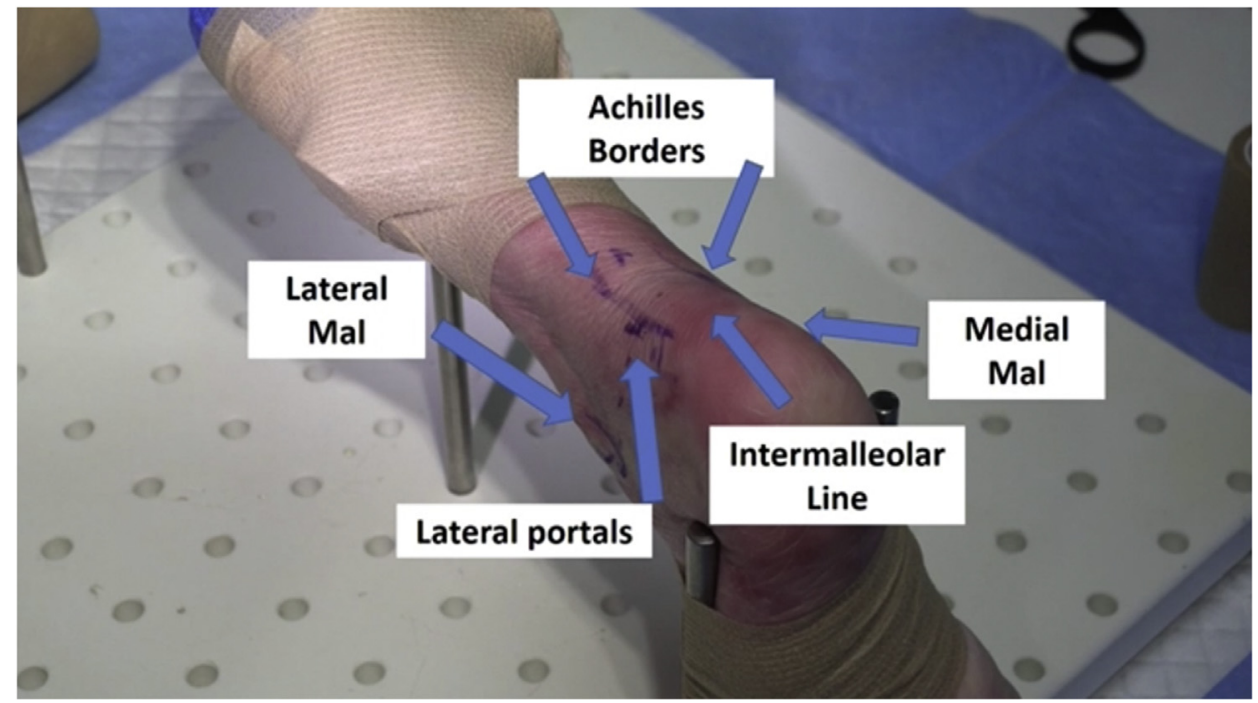

technique in Table 3. The quadrants were divided by the IML horizontally and the center of the ankle vertically. We have preferred to start with the superolateral quadrant and move in a counter-clockwise direction for right ankles and a clockwise direction for left ankles. In the superolateral quadrant, the posterior inferior tibiofibular ligament and IML were identified. If a hypertrophied IML was identified, then it was debrided at this stage. The ankle was passively plantarflexed and dorsiflexed to visualize any impingement of the ligaments. We identified the FHL tendon in the superomedial quadrant through passive flexion and extension of the great toe. All instrumentation was kept in the safe zone lateral to the FHL tendon during the procedure.

The tibiotalar and subtalar joints were examined after resection of the posterior capsule (Figs 5 and 6). We distracted the calcaneus with dorsiflexion of the ankle to assist with full visualization of the tibial plafond and talar dome. Synovitis, hypertrophic capsule, and softtissue impingement were identified and addressed as necessary with a 3.0 resecter. Any bony impingement including Os trigonum and Stieda lesions were burred rather than removed in total, as we were conscious to keep the portal sizes small. The burred bone was simultaneously sucked out through the shaver. Care was taken to calibrate the suction carefully with the inflow from the small diameter IONA, as to avoid a dry field or bubbles interfering with the field of view. This was the only true variation from standard arthroscopic treatment of these pathologies.

Portals were sealed primarily using adhesive wound closure strips (Steri-Strip; 3M, Saint Paul, MN). Sterile dressings and a compression bandage were applied. Patients were allowed to begin weight-bearing as tolerated immediately after surgery with emphasis on

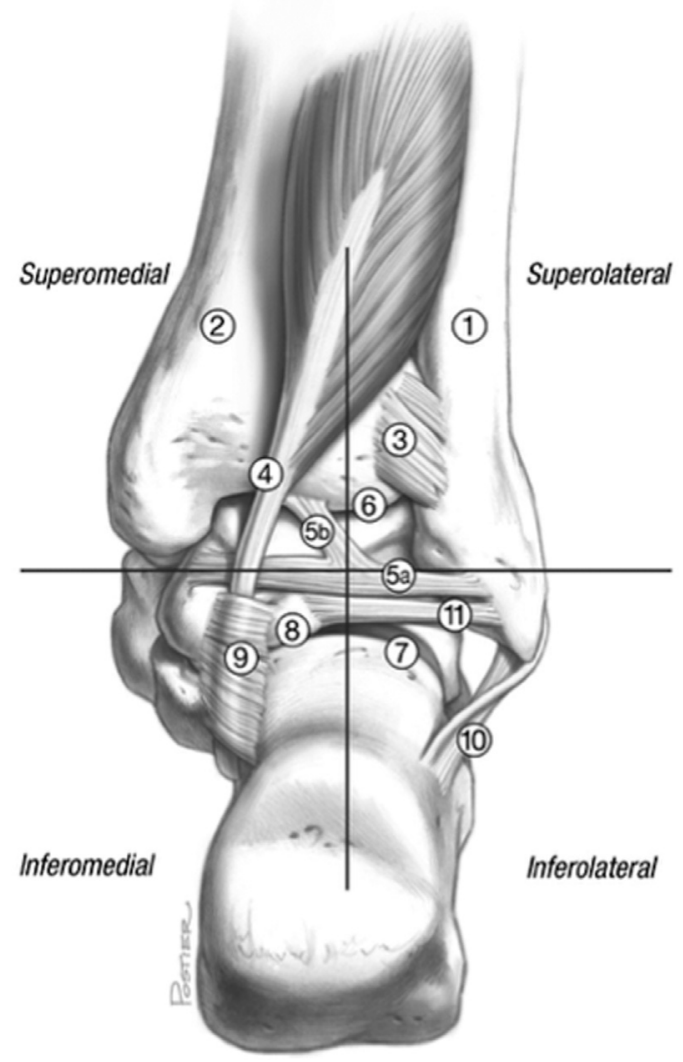

Fig 4. Four-quadrant technique for extra-articular hindfoot structures as defined by the intermalleolar ligament demonstrated on a right ankle. (1) Fibula. (2) Tibia. (3) Posterior inferior tibiofibular ligament. (4) Flexor hallucis longus tendon. (5a) Intermalleolar ligament. (5b) Superior tibial insertion of the intermalleolar ligament. (6) Tibiotalar joint. (7) Subtalar joint. (8) Posterolateral talar process. (9) Flexor hallucis longus retinaculum. (10) Calcaneofibular ligament. (11) Posterior talofibular ligament. Illustration copyright of and reproduced with permission from J. G. Kennedy, M.D. (From Smyth et al. ${ }^{9}$ ) 
Table 3. Pearls and Pitfalls of the In-Office Needle Arthroscopy Technique

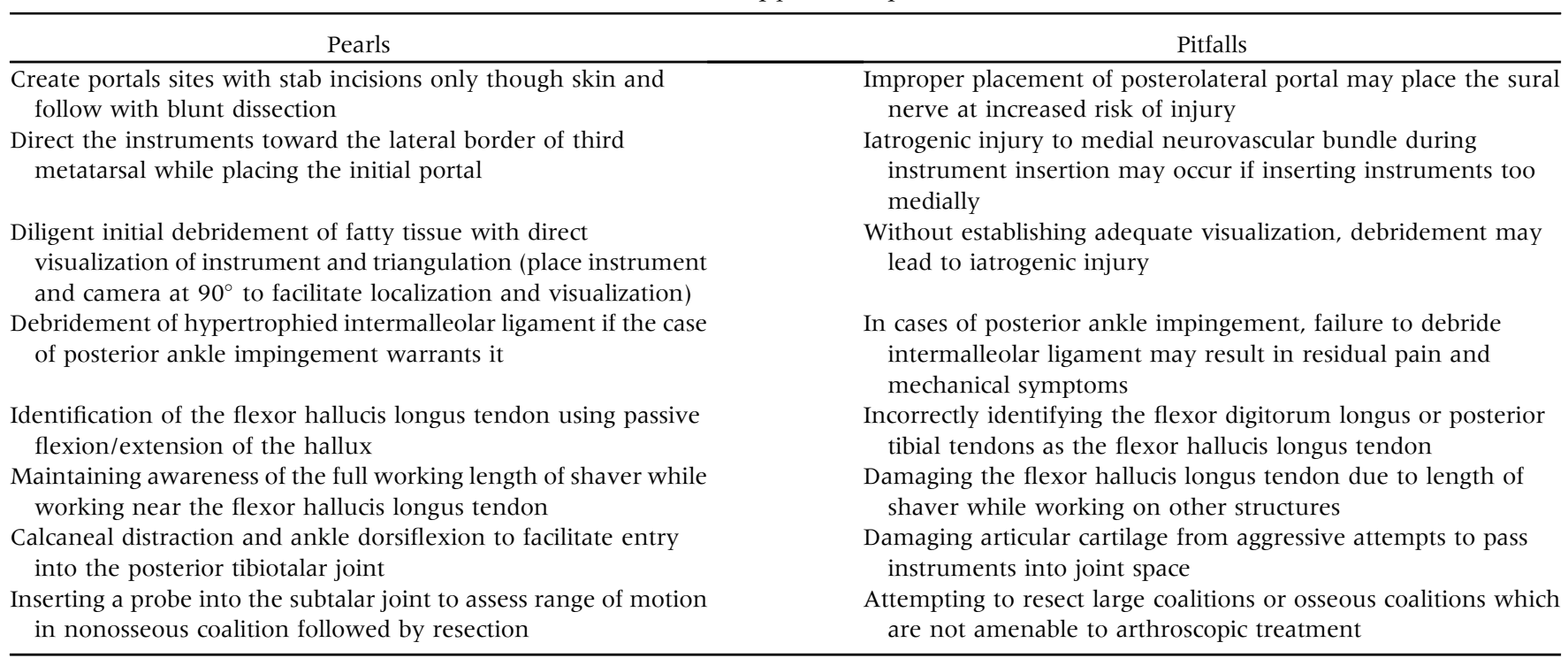

ranging their ankle in dorsiflexion and plantar flexion. However, treatment of significant osseous injury may require protocol modifications based on surgeon discretion. Patients were instructed to perform ankle pumps and circumduction exercises for every hour for 5 minutes for the first 24 hours postoperatively. Ice and elevation were encouraged while at rest. The first follow-up visit was conducted on postoperative day 5 . We instructed all patients to begin physical therapy anywhere from 7 to 10 days after the date of their procedure. The duration of physical therapy was variable, but by 4 weeks' postprocedure most patients were expected to be back at their desired sport.

\section{Results}

\section{Patient Demographics}

In total, 10 patients who underwent IONA for the treatment of posterior ankle impingement in the office setting were identified. All 10 were included in this study, which included 4 male and 6 female patients. The mean age was $41.9 \pm 15.5$ years (range, 24-66 years) and mean body mass index was $28.3 \pm 6.3$ (range, 28.3-39.9). The mean follow-up time was $13.3 \pm 2.9$ months (range, 11-17 months) (Table 4). Eight of the 10 patients had previous surgery related to their PAIS. The mean time from this previous surgery to the IONA procedure was $22.1 \pm 24.0$ months (range, 2-72 months).

\section{Clinical Outcomes}

All evaluated preoperative FAOS mean scores significantly increased at follow-up after IONA, including FAOS symptoms $(71.48 \pm 10.3$ to $80.3 \pm 12.6)$, pain $(69.3 \pm 11.0$ to $78.2 \pm 13.9)$, activities of daily living $(61.7 \pm 8.8$ to $77.93 \pm 11.4)$, sports activities $(55.6 \pm$
12.7 to $76.0 \pm 13.6)$, and quality of life $(46.6 \pm 9.2$ to $71.1 \pm 12.1$ ) (all, $P<.001$ ) (Table 2). The mean PROMIS Pain Intensity T-score significantly decreased from a mean of $57.5 \pm 8.4$ preoperatively to a mean of $49.5 \pm 5.5$ at the final follow-up visit $(P<.001)$. The mean PROMIS Pain Interference T-score significantly decreased from $69.0 \pm 5.8$ preoperatively to $63.1 \pm 5.8$ at the final follow-up visit $(P<.001)$ (Table 2$)$.

\section{Return to Work and Sport}

All patients who worked before IONA procedure returned to regular work activity. The median time to return to work was 3.4 days (range, 0-14 days). There were 7 patients who participated in sports activity before IONA procedure. From this group, all patients

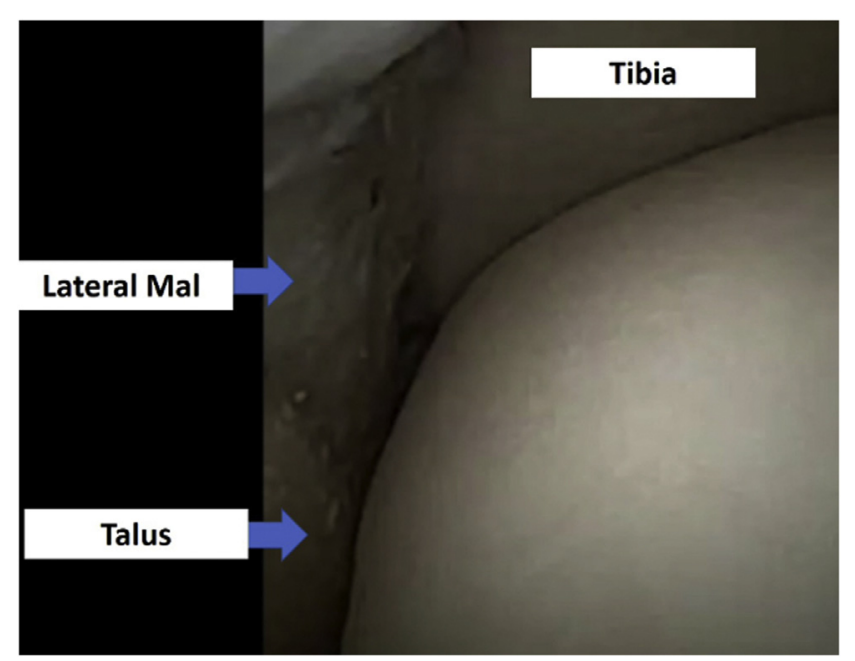

Fig 5. Trifurcation of tibial plafond, talar dome, and lateral malleolus of the tibiotalar joint of a left ankle, a typical location for osteochondral lesions. 


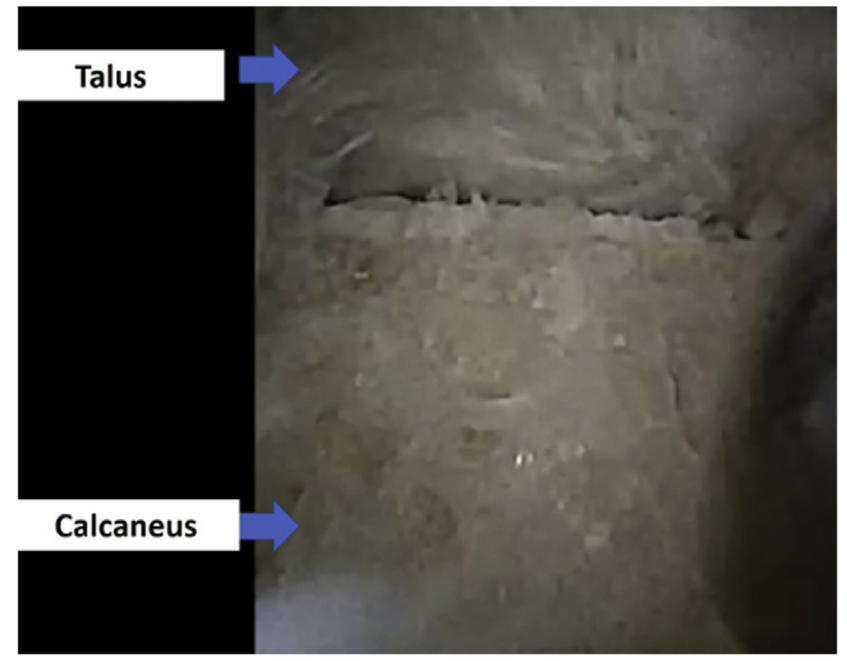

Fig 6. Extra-articular view of subtalar joint of left ankle.

$(100 \%)$ returned to their sports activities with 5 patients $(71 \%)$ returning to the pre-PAIS play level. The mean time to return to sports was 4.8 weeks (range, 214 weeks) (Table 5).

\section{Needle Arthroscopy Experience in the Office Setting}

Patients reported an overall positive experience after IONA, with a mean rating scale of $9.5 \pm 1.5$ (range, 5$10)$. Nine patients $(90 \%)$ reported the highest rating $(10 / 10)$ for overall positive experience and felt that seeing their procedure in real time aided in their understanding of their underlying pathology. Similarly, 8 patients $(80 \%)$ reported the highest rating $(10 / 10)$ for preferring to undergo their procedure in the office setting as opposed to the operating room under

Table 4. Patient Demographics and Characteristics $(\mathrm{n}=10)$

\begin{tabular}{lc}
\hline Age, y & $41.9 \pm 15$ \\
Sex, males/females, n & $4 / 6$ \\
BMI & $28.3 \pm 6.3$ \\
PAIS location, n (\%) & $6(60)$ \\
$\quad$ Right & $4(40)$ \\
$\quad$ Left & $13.3 \pm 2.9$ \\
Follow-up time, mo & 3 \\
History of previous surgery & 1 \\
$\quad$ Os trigonum resection & 1 \\
ORIF of ankle & 1 \\
Subtalar arthrodesis & 1 \\
Loose body removal/tibial exostectomy & 2 \\
ATFL reconstruction/arthroscopy for AMI & $22.1 \pm 24.0$ \\
Previous IONA for PAIS & \\
None & \\
Time from previous surgery to IONA, mo & \\
\hline AMI, anteriomedial impingement; ATFL, anterior talofibular liga- \\
ment; BMI, body mass index; IONA, in-office needle arthroscopy; \\
ORIF, open reduction internal fixation; PAIS, posterior ankle \\
impingement syndrome. \\
*Data are shown as mean \pm standard deviation unless otherwise \\
indicated.
\end{tabular}

Table 5. Return to Play and Return to Work

\begin{tabular}{lc}
\hline & Value \\
\hline Sport activity before IONA, n (\%) & $7(70)$ \\
Return to sports, n (\%) & $7(100)$ \\
Return to same level of play, n (\%) & $5(71)$ \\
Time to return to sports, wk & $48 \pm 3.9$ \\
Working prior to IONA, n (\%) & $7(70)$ \\
Return to work, n (\%) & $7(100)$ \\
Time to return to work, d & $3.4 \pm 5.4$ \\
\hline
\end{tabular}

IONA, in-office needle arthroscopy.

sedation with an overall mean rating of $9.0 \pm 2$ (range, 5-10). The mean satisfaction rating was $4.7 \pm 0.5$ (range, $4-5)$. Seven patients $(70 \%)$ were very satisfied, and 3 patients $(30 \%)$ were satisfied. No patients were neutral, unsatisfied, or very unsatisfied with their outcome. Overall, patients experienced minimal pain with a mean pain rating scale of $0.1 \pm 0.3$ (range, $0-1$ ). Lastly, all patients $(100 \%)$ expressed willingness to undergo the same procedure in the future (Table 6).

\section{Complications}

There were no complications in our patient cohort.

\section{Discussion}

The most important finding of this study is that IONA treatment for PAIS results in significant pain reduction, a low complication rate and excellent patient-reported outcomes. In addition, IONA for PAIS leads to high patient satisfaction with a significant willingness to undergo the same procedure again.

The outcomes of this small case series analyzing IONA for PAIS are promising and may translate to similar results in larger cohort studies in the future. Due to the smaller-sized instrumentation of IONA, common foot

Table 6. Questionnaire Responses $(\mathrm{n}=10)$

\begin{tabular}{lr}
\hline \multicolumn{1}{c}{ Question } & Mean $\pm \mathrm{SD}$ \\
\hline $\begin{array}{l}\text { How was your overall experience seeing } \\
\text { your underlying pathology at the time } \\
\text { of the procedure? }\end{array}$ & $9.5 \pm 1.5$ \\
$\begin{array}{l}\text { Do you feel this experience aided in your } \\
\text { understanding of posterior ankle }\end{array}$ & $10.0 \pm 0$ \\
$\quad$ impingement? & \\
$\begin{array}{l}\text { Would you prefer having this same } \\
\text { procedure in the office, operating }\end{array}$ & $9.0 \pm 2.0$ \\
$\quad$ room, or do you have no preference? & \\
$\begin{array}{l}\text { Did you have any pain during the } \\
\text { procedure? }\end{array}$ & \\
$\begin{array}{l}\text { Likert scale (1-5) } \\
\text { Would you undergo the same procedure } \\
\text { again? (\% yes) }\end{array}$ & $0.1 \pm 0.3$ \\
\end{tabular}

SD, standard deviation.

${ }^{*} 1$-negative experience, 5-neutral, 10-positive experience.

${ }^{\dagger} 1$-operating room, 5-neutral, 10-office.

${ }^{\ddagger} 1$-no pain, 10 -worst pain. 
and ankle pathology can be treated under local anesthesia. The availability of this technology at the time of the COVID-19 pandemic has accelerated its use in the office setting. The purpose of this study was to report the outcomes and draw attention to a procedure that may change future practice guidelines for the treatment of pathologies that do not require complex reconstruction. IONA interventions in our case series involved the removal of pathologic bone and or soft tissue.

Our patients achieved successful clinical outcomes at a mean follow-up time of $13.3 \pm 2.9$ months, with mean PROMIS scores and FAOS scores significantly improving from their preoperative values. We also observed a $100 \%$ return to sport and work in our participating patients. These results mirror published studies for PAIS treated with arthroscopic intervention. Scholten et al. ${ }^{12}$ evaluated 55 consecutive patients with PAIS who received arthroscopic debridement with a median follow-up duration of 36 months. The authors reported a median AOFAS hindfoot score increase from 75 points preoperatively to 90 points postoperatively. Calder et al. ${ }^{6}$ reported on 27 elite professional soccer players with PAIS who received posterior ankle arthroscopy at a mean follow-up time of 23 months, where arthroscopy was used to perform a soft-tissue debridement with flexor hallucis longus debridement, excision of a symptomatic os trigonum, or removal of a bony fragments. In their cohort, the mean length of time to return to training was 34 days and return to playing was 41 days. In our smaller cohort, mean time of return to sports was 4.8 weeks or roughly 34 days. Similarly, Sugimoto et al. ${ }^{13}$ reported on 59 patients who underwent posterior ankle arthroscopy for the treatment of PAIS with a mean follow-up period of 60 months and found that the average time taken to resume training was 5.3 weeks. They also found that the time to return to play was shorter in elite athletes compared with local athletes. Our study cohort did not consist of elite athletes but the return to sport time was shorter than the participants in the study by Sugimoto et al. ${ }^{13}$ This might be potentially attributable to the smaller portal size and less associated softtissue trauma from smaller instruments, which may facilitate an earlier return to sport. Miyamoto et al. ${ }^{14}$ performed simultaneous anterior ankle arthroscopy and hindfoot endoscopy for 9 athletes with impingement symptoms. Mean outcome scores and range of motion improved from preoperative values with a median 12 weeks reported for return to sport. Finally, Georgiannos and Bisbinas ${ }^{15}$ compared endoscopic versus open treatment for PAIS secondary to symptomatic os trigonums as part of a randomized controlled trial. Patients who underwent the endo- scopic procedure had a quicker return to training at a mean of 4.58 weeks versus 9.58 weeks and previous sports level at mean 7.12 weeks versus 11.54 weeks, respectively, when compared with patients who had received the open procedure. The endoscopic procedure was also associated with a lower complication rate.

Our results are promising in our study population and may indicate favorable outcomes in larger studies comparing IONA with traditional arthroscopy for posterior debridement. Our results show that in our patient cohort return to sports after IONA are comparable with the cited rates for traditional arthroscopic treatment of PAIS. Although not a generalizable conclusion, given our study design and population, this finding emphasizes that there is potential utility for IONA in the treatment of posterior hindfoot pathologies.

Wide-awake local anesthesia with no tourniquet (WALANT) procedures have demonstrated multiple benefits over similar traditional operating room interventions. One study reported lower visual analog scale pain scores 1 day after the procedure, shorter hospital stays, fewer total days of oral analgesic use, and fewer missed total working days for patients who underwent open reduction and internal fixation for a distal radius fracture under WALANT when compared with general anesthesia and intravenous regional anesthesia. ${ }^{16}$ WALANT did have a greater mean blood loss versus general anesthesia (23.4 mL vs $11.5 \mathrm{~mL}$ ); however, this difference was likely not clinically significant. Furthermore, WALANT procedures do not require an anesthesia team, operating room staff, or extended postoperative recovery time, which all lower the direct clinical costs when compared with a full operating suite. As demonstrated by our own results, patient satisfaction is also generally high after WALANT procedures. We expect WALANT procedures to continue growing in popularity as technology continues to advance and indications are better defined.

IONA for the diagnosis and intervention of foot and ankle pathology has already been demonstrated to be a safe tool in cadaver models. ${ }^{17,18}$ We have confirmed these initial observations by demonstrating that select patients with PAIS can be successfully managed under WALANT conditions with results comparable to endoscopy in a full operating room suite. We stress the importance of having a thorough conversation with the patient and understanding his or her pathology before the procedure to ensure that the indications for IONA are met. When done correctly, IONA offers a unique opportunity for the patient to learn more about his or her pathology in real-time, facilitating an understanding of expectations of rehabilitation and ultimately accelerating both return to sport as well as 
to work. We expect future studies to confirm the effectiveness of IONA for treating additional musculoskeletal pathologies with comparisons to established techniques.

\section{Limitations}

Our study had several important limitations. First, our study was a retrospective analysis, which may introduce recall bias as patients described their previous work and sports involvement. Second, the low number of patients involved in the study may not appropriately represent the true results from treating PAIS with IONA and limits the ability to generalize our results to all patients. Next, our shorter follow-up time may have missed later recurrence of PAIS symptoms. In addition, we acknowledge that not all pathology causing PAIS can be managed by IONA. By choosing IONA for our patients, we undertook small os trigonum resection and soft-tissue resections initially. With each subsequent IONA procedure for PAIS, the authors cumulatively progressed along the learning curve and outcomes and confidence improved. We began performing larger Stieda process removals as the series evolved and our learning curve accelerated. Therefore, the progression of our experience with this treatment modality may have impacted the outcomes of our patient cohort. Lastly, a potential limitation of our study may be the possibility of selection bias within our cohort of patients, and thus there may have been a bias in the patient population that elected to receive this intervention. Larger studies with an extended followup period will be necessary to fully evaluate IONA for all types of PAIS.

\section{Conclusions}

The current study demonstrates that IONA treatment of PAIS results in significant pain reduction, a low complication rate, and excellent patient-reported outcomes. Additionally, IONA for PAIS leads to high patient satisfaction with a significant willingness to undergo the same procedure again.

\section{References}

1. Yasui Y, Hannon CP, Hurley E, Kennedy JG. Posterior ankle impingement syndrome: A systematic four-stage approach. World J Orthop 2016;7:657-663. doi:10.5312/ wjo.v7.il 0.657.

2. McAlister JE, Urooj U. Os trigonum syndrome. Clin Podiatr Med Surg 2021;38:279-290. doi:10.1016/j.cpm.2020.12. 011.

3. Zwiers R, Wiegerinck JI, Murawski CD, Smyth NA, Kennedy JG, van Dijk CN. Surgical treatment for posterior ankle impingement. Arthroscopy 2013;29:1263-1270. doi: 10.1016/j.arthro.2013.01.029.
4. Smyth NA, Zwiers R, Wiegerinck JI, et al. Posterior hindfoot arthroscopy: A review. Am J Sports Med 2014;42: 225-234. doi:10.1177/0363546513491213.

5. Roche AJ, Calder JD, Lloyd Williams R. Posterior ankle impingement in dancers and athletes. Foot Ankle Clin 2013;18:301-318. doi:10.1016/j.fcl.2013.02. 008.

6. Calder JD, Sexton SA, Pearce CJ. Return to training and playing after posterior ankle arthroscopy for posterior impingement in elite professional soccer. $A m \mathrm{~J}$ Sports Med 2010;38:120-124. doi:10.1177/ 0363546509346390.

7. Zhang K, Crum RJ, Samuelsson K, Cadet E, Ayeni OR, de Sa D. In-office needle arthroscopy: A systematic review of indications and clinical utility. Arthroscopy 2019;35: 2709-2721. doi:10.1016/j.arthro.2019.03.045.

8. Moletta L, Pierobon ES, Capovilla G, et al. International guidelines and recommendations for surgery during Covid-19 pandemic: A systematic review. Int J Surg 2020;79:180-188. doi:10.1016/j.ijsu.2020.05.061.

9. Smyth NA, Murawski CD, Levine DS, Kennedy JG. Hindfoot arthroscopic surgery for posterior ankle impingement: A systematic surgical approach and case series. Am J Sports Med 2013;41:1869-1876.

10. Harris JD, Brand JC, Cote MP, Faucett SC, Dhawan A. Research pearls: The significance of statistics and perils of pooling. Part 1: Clinical versus statistical significance. Arthroscopy 2017;33:1102-1112. doi:10.1016/j.arthro. 2017.01 .053$.

11. Sedaghat AR. Understanding the minimal clinically important difference (MCID) of patient-reported outcome measures. Otolaryngol Head Neck Surg 2019;161:551-560. doi:10.1177/0194599819852604.

12. Scholten PE, Sierevelt IN, van Dijk CN. Hindfoot endoscopy for posterior ankle impingement. $J$ Bone Joint Surg Am 2008;90:2665-2672. doi:10.2106/JBJS.F. 00188.

13. Sugimoto K, Isomoto S, Samoto N, Matsui T, Tanaka Y. Arthroscopic treatment of posterior ankle impingement syndrome: Mid-term clinical results and a learning curve. Arthrosc Sports Med Rehabil 2021;3:e1077-e1086. doi:10. 1016/j.asmr.2021.03.013.

14. Miyamoto W, Miki S, Kawano H, Takao M. Surgical outcome of posterior ankle impingement syndrome with concomitant ankle disorders treated simultaneously in patient engaged in athletic activity. J Orthop Sci 2017;22: 463-467. doi:10.1016/j.jos.2016.12.017.

15. Georgiannos D, Bisbinas I. Endoscopic versus open excision of Os trigonum for the treatment of posterior ankle impingement syndrome in an athletic population: A randomized controlled study with 5-year follow-up. Am J Sports Med 2017;45:1388-1394. doi:10.1177/ 0363546516682498.

16. Tahir M, Chaudhry EA, Zaffar Z, et al. Fixation of distal radius fractures using wide-awake local anaesthesia with no tourniquet (WALANT) technique: A randomized control trial of a cost-effective and resource-friendly procedure. Bone Joint Res 2020;9:429-439. doi:10.1302/20463758.97.BJR-2019-0315.R1. 
17. Stornebrink T, Altink JN, Appelt D, Wijdicks CA, Stufkens SAS, Kerkhoffs GMMJ. Two-millimetre diameter operative arthroscopy of the ankle is safe and effective. Knee Surg Sports Traumatol Arthrosc 2020;28: 3080-3086. doi:10.1007/s00167-020-05889-7.
18. Stornebrink T, Emanuel KS, Shimozono Y, Karlsson J, Kennedy JG, Kerkhoffs GMMJ. A change in scope: Redefining minimally invasive. Knee Surg Sports Traumatol Arthrosc 2020;28:3064-3065. doi:10.1007/s00167-02005898-6. 